7-28-2020

\title{
Even Order Ranked Set Sampling with Auxiliary Variable
}

\author{
Muhammad Tayyab \\ National College of Business Administration \& Economics, Lahore, Pakistan, m_tayyab82@hotmail.com \\ Muhammad Noor ul-Amin \\ COMSATS University Islamabad-Lahore Campus Lahore, Pakistan, nooramin.stats@gmail.com \\ Muhammad Hanif \\ National College of Business Administration \& Economics, Lahore, Pakistan, drhanif@ncbae.edu.pk
}

Follow this and additional works at: https://digitalcommons.wayne.edu/jmasm

Part of the Applied Statistics Commons, Social and Behavioral Sciences Commons, and the Statistical Theory Commons

\section{Recommended Citation}

Tayyab, M., Noor ul-Amin, M., Hanif, M. (2019). Even Order Ranked Set Sampling with Auxiliary Variable. Journal of Modern Applied Statistical Methods, 18(2), eP2751. https://doi.org/10.22237/jmasm/ 1604189280

This Regular Article is brought to you for free and open access by the Open Access Journals at DigitalCommons@WayneState. It has been accepted for inclusion in Journal of Modern Applied Statistical Methods by an authorized editor of DigitalCommons@WayneState. 


\section{Even Order Ranked Set Sampling with Auxiliary Variable}

\author{
Muhammad Tayyab \\ National College of Business \\ Administration \& Economics \\ Lahore, Pakistan
}

\author{
Muhammad Noor ul-Amin \\ COMSATS University \\ Islamabad-Lahore Campus \\ Lahore, Pakistan
}

\author{
Muhammad Hanif \\ National College of Business \\ Administration \& Economics \\ Lahore, Pakistan
}

Even order ranked set sampling (EORSS) is a novel proposed ranked set sampling scheme connected with an auxiliary variable correlated with the study variable. This scheme quantifies only the one sampling unit which is at even position from each ranking set by employing specific criteria. The performance of the ratio estimator under EORSS is compared to its contemporary estimators in simple random sampling (SRS), ranked set sampling (RSS), median ranked set sampling (MRSS) and quartile ranked set sampling (QRSS) exploiting the same number of quantified units. The simulation results proved that EORSS is an efficient alternative sampling scheme for ratio estimation than SRS, RSS, MRSS and QRSS.

Keywords: Auxiliary information, even order ranked set sampling, ranked set sampling, ratio estimator

\section{Introduction}

McIntyre (1952) proposed a precise sampling scheme than usual SRS. This idea was popularized as RSS by Takahasi and Wakimoto (1968) with deriving its mathematical results. They demonstrated the mean of ranked set sample as unbiased estimator of population mean having smaller variance than the sample mean obtained from SRS. Dell and Clutter (1972) noticed that errors occurred in ranking cause loss in RSS efficiency. Samawi and Muttlak (1996) estimated the ratio under RSS and endorsed that ranking on auxiliary variable $X$ is more effective than that on study variable $Y$. Samawi (2002) estimated the population ratio employing ERSS and presented that ratio estimator is approximately unbiased. A simulation assessment between different ranked set sampling procedures and SRS are discussed by Ibrahim (2011). Haq et al. (2014) developed a mixed-ranked set

doi: 10.22237/jmasm/1604189280 | Accepted: Mar. 8, 2018; Published: July 28, 2020.

Correspondence: Muhammad Tayyab,m_tayyab82@hotmail.com 


\section{TAYYAB ET AL.}

sampling scheme showing efficient behavior for median and mean estimation. A comprehensive study on RSS and modified RSS methods has been done by Stokes (1977), Samawi and Muttlak (2000), Ahmad et al. (2010), Khan and Shabbir (2016) and Zamanzade and Al-Omari (2015).

Auxiliary information is valuable for specific situations where the actual measurement of the study variable is difficult, pricey or destructive. Information on auxiliary variable is already available alongside the variable of interest. When auxiliary variable is used in estimation of population parameters the performance of estimators are enhanced to a very good extent. Detailed information on auxiliary variable can be found in Bouza (2010), Noor-ul-Amin and Hanif (2012), Sanaullah et al. (2014) and Noor-ul-Amin et al. (2017).

The RSS procedure using ranking on auxiliary variable for estimating the ratio-type estimators is described by Koyuncu (2015) as: $k^{2}$ bivariate elements are chosen randomly from underlying population and distribute these units into $k$ sets at random with set size $k$. The $k$ bivariate units of every set are organized according to auxiliary characteristic $X$ in ascending order without identifying the actual measurements of study variable $Y$. Afterword the minimum $X$ with connected $Y$ are quantified from the first set and the second minimum unit of $X$ with connected unit of $Y$ from second set, so forth until from the $k$ th set of $k$ bivariate units the biggest unit $X$ with connected $Y$ are selected. The entire steps are repeated $\mathrm{r}$ times until the required sample $n=k r$ from initial $k^{2} r$ bivariate units is obtained.

Muttlak (1997) introduced the new sampling technique named median ranked set sampling (MRSS). In this scheme, distribution of $k^{2}$ sampling units in $k$ sets and cycles are totally same as usual RSS and the selection procedure is as follows: If $k$ is odd then $((k+1) / 2)$ th bivariate units from all sets are selected as sample. In case $k$ is even then lowest bivariate units from the two middle measurements of first $k / 2$ sets along with the largest measurements from the two middle values of the remaining $k / 2$ sets are selected as sample.

Muttlak (2003) proposed quartile ranked set sampling (QRSS) and demonstrated the distribution of $k^{2}$ sampling units with $k$ set size and $r$ cycles in the same manner as used in RSS. The selection procedure of QRSS is as follows: If $k$ is odd, pick from first $(k-1) / 2$ ranked sets the $((k+1) / 4)$ th observation and from last $(k-1) / 2$ ranked sets the $(3(k+1) / 4)$ th observation and take the median observation from central set. If $k$ is even, pick from first $k / 2$ sets the $((k+1) / 4)$ th ranked observation and from remaining $k / 2$ sets the $(3(k+1) / 4)$ th ranked observation. The nearest ranked observation of the quartiles is selected in case of decimals. 


\section{EVEN ORDER RANKED SET SAMPLING}

The sampling scheme considering the ranked sets can be improved by utilizing inexpensive auxiliary information about the ranking of the units. In rest of this research article, a new RSS scheme named EORSS by using an auxiliary variable is introduced and expressions for mean squared error (MSE) of the ratio estimators under EORSS are derived. A mixture method in Bayesian context is applied to simulate the non-normal bivariate distributions because $\mathrm{R}$ codes are not available in literature. The utilization of proposed scheme is identified by comparing with some existing rank set sampling schemes. Therefore, a comprehensive simulation study for normal and non-normal bivariate distributions is carried out to compare the performance of EORSS ratio estimator with its counterparts in SRS, RSS, MRSS and QRSS.

\section{Even Order Ranked Set Sampling}

Even order ranked set sampling (EORSS) scheme is proposed to estimate ratio estimator for the population mean. Even order ranked set sample considers only the one sampling unit which is at even position from each ranking set. Sometime the ranking of the investigational units of study variable is pricey or time consuming in the field of ecological, medical and environmental studies. Therefore ranking is executed by means of an auxiliary variable $X$ that can be easily measured with no cost and has positive correlation with the study variable $Y$. The EORSS design is an alternative to the classical RSS that performs better than SRS, RSS, MRSS and QRSS to estimate the ratio estimator for population mean.

A sample based on EORSS can be chosen as:

1. Pick $k^{2}$ bivariate sample units at random from population.

2. Allocate $k^{2}$ selected bivariate sample units into $k$ sets of identical size $k$.

3. Ranking is done with values of $X$ with correlated study variable $Y$. Rank the $k$ bivariate sample units within each set in increasing order without knowing the any measurements of study variable $Y$.

4. If $k$ is even then for the first $k / 2$ sets choose second minimum unit of $X$ with connected unit of $Y$ from first set, fourth minimum unit of $X$ with connected unit $Y$ from second set and so forth $k$ th unit of $X$ with connected unit of $Y$ from $(k / 2)$ th set. Same process use to choose sample units from other $k / 2$ sets for completing the sample size. If $k$ is odd then from first $(k-1) / 2$ sets, choose second minimum unit of $X$ with connected unit of $Y$ from first set, fourth minimum unit of $X$ with 


\section{TAYYAB ET AL.}

connected unit $Y$ from second set and so forth $(k-1)$ th ranked unit of $X$ with connected unit of $Y$ from $((k-1) / 2)$ th set. Same process is adopted to choose sample units from other $(k-1) / 2$ sets and select $(k-1)$ th ranked unit of $X$ with connected unit $Y$ from remaining set for completing the one cycle of the sample size.

5. Repeat the above procedure $r$ cycles, until the required sample size $n=k r$ from initial $k^{2} r$ bivariate sample units is completed.

Assume $\left(X_{i}, Y_{i}\right)(i=1,2,3, \ldots, k)$ symbolize a bivariate normal random sample with pdf $f(x, y)$, cdf $F(x, y)$, population means $\mu_{x}, \mu_{y}$, variances $\sigma_{x}^{2}, \sigma_{y}^{2}$ and correlation $\rho_{x y}$. Suppose ranking is done on auxiliary variable $X$ to estimate the mean of study variable $Y$, let $\left(X_{(i)}, Y_{[i]}\right)$ indicate the $i$ th order statistic of the $i$ th set for the auxiliary variable and $i$ th judgment ordering for $i$ th set of study variable $Y$.

Let $\left(X_{i j}, Y_{i j}\right), i, j=1,2,3, \ldots, k$, denote the $k$ independent bivariate normal random vectors of same size $k$ and $\left(X_{1(1) c}, Y_{1[1] c}\right),\left(X_{2(2) c}, Y_{2[2] c}\right), \ldots,\left(X_{k(k) c}, Y_{k[k] c}\right)$ represent the ranked set sample. Assume $\left(X_{1(2) c}, Y_{1[2] c}\right),\left(X_{2(4) c}, Y_{2[4] c}\right), \ldots$, $\left(X_{\frac{k}{2}(k) c}, Y_{\frac{k}{2}[k] c}\right),\left(X_{\frac{k}{2}+1(2) c}, Y_{\frac{k}{2}+1[2] c}\right),\left(X_{\frac{k}{2}+2(4) c}, Y_{\frac{k}{2}+2[4] c}\right), \ldots,\left(X_{k(k) c}, Y_{k[k] c}\right)$ be the EORSS for even sample size, and $\left(X_{1(2) c}, Y_{1[2] c}\right),\left(X_{2(4) c}, Y_{2[4] c}\right), \ldots,\left(X_{\frac{k-1}{2}(k-1) c}, Y_{\frac{k-1}{2}[k-1] c}\right)$, $\left(X_{\frac{k-1}{2}+1(2) c}, Y_{\frac{k-1}{2}+1[2] c}\right),\left(X_{\frac{k-1}{2}+2(4) c}, Y_{\frac{k-1}{2}+2[4] c}\right), \ldots,\left(X_{k-1(k-1) c}, Y_{k-1[k-1] c}\right),\left(X_{k(k-1) c}, Y_{k[k-1] c}\right)$ be the EORSS for odd sample size, where $c=1,2,3, \ldots, r$.

The respective sample mean of auxiliary variable $X$ and study variable $Y$ using EORSS with even sample size $k$ is defined

$$
\begin{gathered}
\bar{X}_{(O) e}=\frac{1}{k r} \sum_{c=1}^{r}\left[\sum_{i=1}^{\frac{k}{2}} X_{i(2 i: k) c}+\sum_{i=1}^{\frac{k}{2}} X_{\frac{k}{2}+i(2 i: k) c}\right] \\
\bar{Y}_{[O]_{e}}=\frac{1}{k r} \sum_{c=1}^{r}\left[\sum_{i=1}^{\frac{k}{2}} Y_{i[2 i: k] c}+\sum_{i=1}^{\frac{k}{2}} Y_{\frac{k}{2}+i[2 i: k] c}\right]
\end{gathered}
$$

The variances and covariance expressions are obtained by using the results from Dell and Clutter (1972) and Arnold et al. (1992). From (1) and (2)

$$
V\left(\bar{X}_{(o) e}\right)=\frac{2}{r k^{2}}\left[\sum_{i=1}^{\frac{k}{2}} \sigma_{x(2 i: k)}^{2}\right]
$$




\section{EVEN ORDER RANKED SET SAMPLING}

$$
\begin{gathered}
V\left(\bar{Y}_{[O]_{e}}\right)=\frac{2}{r k^{2}}\left[\sum_{i=1}^{\frac{k}{2}} \sigma_{y[2 i k]}^{2}\right] \\
\operatorname{COV}\left(\bar{X}_{(o)_{e}}, \bar{Y}_{[o]_{e}}\right)=\beta V\left(\bar{X}_{(o)_{e}}\right)
\end{gathered}
$$

The respective sample mean of auxiliary variable $X$ and study variable $Y$ using EORSS with odd sample size $k$ is defined

$$
\begin{gathered}
\bar{X}_{(O)_{o}}=\frac{1}{k r} \sum_{c=1}^{r}\left[\sum_{i=1}^{(k-1) / 2} X_{i(2 i k) c}+\sum_{i=1}^{(k-1) / 2} X_{\frac{k-1}{2}+(2 i \cdot i k) c}+X_{k(k-1: k) c}\right] \\
\bar{Y}_{[o]_{o}}=\frac{1}{k r} \sum_{c=1}^{r}\left[\sum_{i=1}^{(k-1) / 2} Y_{i[2 i k] c}+\sum_{i=1}^{(k-1) / 2} Y_{\frac{k-1}{2}+i[2 i k \cdot] c}+Y_{k[k-1: k] c}\right]
\end{gathered}
$$

By using (6) and (7), variances and covariance expressions are derived and given by

$$
\begin{gathered}
V\left(\bar{X}_{(o) o}\right)=\frac{2}{r k^{2}}\left[\sum_{i=1}^{(k-1) / 2} \sigma_{x(2 i k)}^{2}\right]+\frac{1}{k^{2}}\left[\sigma_{x(k-1: k)}^{2}\right] \\
V\left(\bar{Y}_{[O]_{o}}\right)=\frac{2}{r k^{2}}\left[\sum_{i=1}^{(k-1) / 2} \sigma_{y[2 i k]}^{2}\right]+\frac{1}{k^{2}}\left[\sigma_{y[k-1: k]}^{2}\right] \\
\operatorname{COV}\left(\bar{X}_{(o)_{o}, \bar{Y}_{[o]_{o}}}\right)=\beta V\left(\bar{X}_{(o)_{o}}\right)
\end{gathered}
$$

The ratio estimator based on EORSS can be defined

$$
\hat{\mu}_{y o h}=\mu_{x} \frac{\bar{Y}_{[o] h}}{\bar{X}_{(o) h}}
$$

Using bivariate Taylor series as 


$$
\begin{aligned}
& f\left(\bar{X}_{(o) h}, \bar{Y}_{[o] h}\right) \cong f\left(\mu_{x}, \mu_{y}\right)+\left(\bar{X}_{(o) h}-\mu_{x}\right) f_{\bar{X}_{(o) h}}\left(\mu_{x}, \mu_{y}\right) \\
& +\left(\bar{Y}_{[o] h}-\mu_{y}\right) f_{\overline{\bar{Y}}_{[o] h}}\left(\mu_{x}, \mu_{y}\right) \\
& +\frac{1}{2 !}\left[\begin{array}{l}
\left(\bar{X}_{(o) h}-\mu_{x}\right)^{2} f_{\bar{X}_{(o) h} \bar{X}_{(o) h}}\left(\mu_{x}, \mu_{y}\right) \\
+\left(\bar{Y}_{[o] h}-\mu_{y}\right)^{2} f_{\bar{Y}_{[o j h} \bar{T}_{o]_{h}}}\left(\mu_{x}, \mu_{y}\right) \\
+2\left(\bar{X}_{(o) h}-\mu_{x}\right)\left(\bar{Y}_{[o] h}-\mu_{y}\right) f_{\bar{X}_{(o) h} \bar{T}_{[o] h}}\left(\mu_{x}, \mu_{y}\right)
\end{array}\right]
\end{aligned}
$$

where $f\left(\bar{X}_{(o) h}, \bar{Y}_{[o] h}\right)=\hat{\mu}_{y O h}$ and $h=e, o$ denotes the sample size is even or odd. By using (12), the first-degree of approximation of the estimator in (11) can be obtained as

$$
\hat{\mu}_{y O h}=\mu_{y}+\bar{Y}_{[o] h}-\mathrm{R} \bar{X}_{(o) h}
$$

where $\mathrm{R}=\frac{\mu_{y}}{\mu_{x}}$, the MSE of $\hat{\mu}_{y O h}$ from (13) is approximated as

$$
\begin{aligned}
\operatorname{MSE}\left(\hat{\mu}_{y O h}\right) \cong V\left(\bar{Y}_{[O]_{h}}\right)+\mathrm{R}^{2} V\left(\bar{X}_{(o) h}\right)-2 \mathrm{R} C O V\left(\bar{X}_{(o) h}, \bar{Y}_{[O]_{h}}\right) \\
\cong\left\{\begin{array}{l}
\frac{2}{r k^{2}} \sum_{i=1}^{\frac{k}{2}}\left[\sigma_{y[2 i k]}^{2}-\left(2 \mathrm{R} \beta-\mathrm{R}^{2}\right) \sigma_{x(2 i k)}^{2}\right], \quad k \text { is even } \\
\frac{2}{r k^{2}} \sum_{i=1}^{(k-1) / 2}\left[\sigma_{y[2 i k]}^{2}-\left(2 \mathrm{R} \beta-\mathrm{R}^{2}\right) \sigma_{x(2 i k)}^{2}\right] \\
+\frac{1}{r k^{2}}\left[\sigma_{y[k-1: k]}^{2}-\left(2 \mathrm{R} \beta-\mathrm{R}^{2}\right) \sigma_{x(k-1: k)}^{2}\right], \quad k \text { is odd }
\end{array}\right.
\end{aligned}
$$

\section{Performance Evaluation and Comparisons}

A simulation process is conducted to examine the performance of ratio estimator for the population mean using SRS, RSS, MRSS, QRSS and suggested improved sampling design EORSS. The random samples are drawn from three bivariate distributions; bivariate-normal, bivariate-beta and bivariate-gamma. The bivariate normal random samples are generated from distribution $N(2,2,1,1, \rho)$ where $\rho=0.4,0.6,0.8,0.9$. The efficiency of RSS, MRSS, QRSS and proposed EORSS 


\section{EVEN ORDER RANKED SET SAMPLING}

ratio estimators with respect to SRS ratio estimator are obtained on the basis of 60,000 repetitions and presented in Table 1 .

Table 1. The efficiency of the ratio estimator for RSS, MRSS, QRSS and EORSS relating to the SRS from bivariate-normal with $\mu_{x}=\mu_{y}=2, \sigma_{x}^{2}=\sigma_{y}^{2}=1$ along with different set size and $\rho$.

\begin{tabular}{cccccccc}
$\boldsymbol{\rho}$ & $\boldsymbol{k}$ & $\mathbf{5}$ & $\mathbf{6}$ & $\mathbf{7}$ & $\mathbf{8}$ & $\mathbf{9}$ & $\mathbf{1 0}$ \\
\hline \multirow{4}{*}{$\mathbf{0 . 9}$} & RSS & 1.188228 & 1.167005 & 1.133967 & 1.140695 & 1.137626 & 1.128035 \\
& MRSS & 1.218790 & 1.174805 & 1.158316 & 1.148228 & 1.140720 & 1.132308 \\
& QRSS & 1.206308 & 1.171899 & 1.150512 & 1.138661 & 1.130743 & 1.133688 \\
& EORSS & 1.333546 & 1.548959 & 1.291153 & 1.426882 & 1.259793 & 1.362031 \\
\hline \multirow{4}{*}{$\mathbf{0 . 8}$} & RSS & 1.302307 & 1.238017 & 1.222453 & 1.195774 & 1.193590 & 1.166182 \\
& MRSS & 1.330987 & 1.260618 & 1.244655 & 1.210337 & 1.204686 & 1.191555 \\
& QRSS & 1.324981 & 1.238161 & 1.204485 & 1.192388 & 1.205796 & 1.186260 \\
& EORSS & 1.484765 & 1.644723 & 1.360944 & 1.509686 & 1.336128 & 1.413278 \\
\hline \multirow{4}{*}{$\mathbf{0 . 6}$} & RSS & 1.429923 & 1.427700 & 1.341239 & 1.355854 & 1.326339 & 1.311629 \\
& MRSS & 1.481953 & 1.454711 & 1.372448 & 1.380037 & 1.362534 & 1.338390 \\
& QRSS & 1.469308 & 1.443207 & 1.356576 & 1.345432 & 1.329448 & 1.321199 \\
& EORSS & 1.642253 & 1.916956 & 1.525443 & 1.656588 & 1.498317 & 1.588022 \\
\hline \multirow{4}{*}{$\mathbf{0 . 4}$} & RSS & 1.625997 & 1.518060 & 1.519059 & 1.461134 & 1.442210 & 1.468160 \\
& MRSS & 1.715894 & 1.593497 & 1.580553 & 1.490383 & 1.499229 & 1.501665 \\
& QRSS & 1.688733 & 1.545757 & 1.560290 & 1.483212 & 1.497520 & 1.497406 \\
& EORSS & 1.897007 & 2.058115 & 1.745815 & 1.829253 & 1.650347 & 1.770430 \\
\hline
\end{tabular}

A bivariate normal distribution is easy to simulate as $\mathrm{R}$ codes are available in literature. In practice, sometimes statistical bivariate distributions are shown nonnormal or skewed behavior and their R codes do not exist in literature. For nonnormal data, Michael and Schucany (2002) introduced a convenient simulation procedure to simulate bivariate distributions, named as mixture method for bivariate distributions with specific correlations. To generate bivariate pair of observations $(x, y)$, the mixture approach algorithm has the following steps:

Step 1: $\quad$ Specify the prior distribution and generate simulated values, $x$, from prior $\operatorname{pdf} r(x)$.

Step 2: $\quad$ Formulate likelihood conditioned upon $x$ and generate the data, $u$, from likelihood $s(u \mid x)$.

Step 3: Generate simulated values, $y$, from derived posterior distribution $t(y \mid u)$. 


\section{TAYYAB ET AL.}

Table 2. The efficiency of the ratio estimator for RSS, MRSS, QRSS and EORSS relating to the SRS from bivariate-beta $(3,3)$ with different set size and $\rho$.

\begin{tabular}{cccccccc}
$\boldsymbol{\rho}$ & $\boldsymbol{k}$ & $\mathbf{5}$ & $\mathbf{6}$ & $\mathbf{7}$ & $\mathbf{8}$ & $\mathbf{9}$ & $\mathbf{1 0}$ \\
\hline \multirow{4}{*}{$\mathbf{0 . 9}$} & RSS & 1.102594 & 1.111557 & 1.076606 & 1.101753 & 1.081735 & 1.090704 \\
& MRSS & 0.989061 & 0.984640 & 0.956396 & 0.951320 & 0.978945 & 0.950945 \\
& QRSS & 1.038799 & 1.072861 & 1.035877 & 1.098765 & 1.012179 & 1.051570 \\
& EORSS & 1.135290 & 1.342427 & 1.115867 & 1.298422 & 1.131475 & 1.231094 \\
\hline \multirow{4}{*}{$\mathbf{0 . 8}$} & RSS & 1.124028 & 1.166836 & 1.131330 & 1.124093 & 1.111835 & 1.099655 \\
& MRSS & 1.068345 & 1.088283 & 1.088833 & 1.014447 & 0.959431 & 1.037502 \\
& QRSS & 1.083815 & 1.124725 & 1.113888 & 1.116846 & 1.053671 & 1.054277 \\
& EORSS & 1.194136 & 1.410437 & 1.202430 & 1.336537 & 1.184566 & 1.243572 \\
\hline \multirow{4}{*}{$\mathbf{0 . 6}$} & RSS & 1.227834 & 1.238197 & 1.314849 & 1.299120 & 1.242575 & 1.205179 \\
& MRSS & 1.195580 & 1.154226 & 1.242134 & 1.255797 & 1.177213 & 1.220555 \\
& QRSS & 1.199493 & 1.194837 & 1.311787 & 1.285738 & 1.197220 & 1.168033 \\
& EORSS & 1.301698 & 1.525097 & 1.438354 & 1.541549 & 1.307186 & 1.363338 \\
\hline \multirow{2}{*}{$\mathbf{0 . 4}$} & RSS & 1.341488 & 1.362567 & 1.359939 & 1.351739 & 1.342450 & 1.350697 \\
& MRSS & 1.266464 & 1.263278 & 1.249188 & 1.245712 & 1.251505 & 1.226154 \\
& QRSS & 1.284900 & 1.352532 & 1.343158 & 1.338290 & 1.291587 & 1.317633 \\
& EORSS & 1.405613 & 1.665289 & 1.451405 & 1.590239 & 1.460297 & 1.528569 \\
\hline
\end{tabular}

Table 3. The efficiency of the ratio estimator for RSS, MRSS, QRSS and EORSS relating to the SRS from bivariate-gamma $(2,3)$ with different set size and $\rho$.

\begin{tabular}{cccccccc}
$\boldsymbol{\rho}$ & $\boldsymbol{k}$ & $\mathbf{5}$ & $\mathbf{6}$ & $\mathbf{7}$ & $\mathbf{8}$ & $\mathbf{9}$ & $\mathbf{1 0}$ \\
\hline & RSS & 1.132454 & 1.120081 & 1.101168 & 1.088684 & 1.076618 & 1.045625 \\
$\mathbf{0 . 9}$ & MRSS & 0.984479 & 0.983218 & 0.926360 & 0.926182 & 0.966656 & 0.941802 \\
& QRSS & 1.050532 & 1.084240 & 1.048778 & 1.072345 & 1.012312 & 1.064821 \\
& EORSS & 1.149154 & 1.302834 & 1.119297 & 1.285764 & 1.098900 & 1.154353 \\
\hline \multirow{4}{*}{$\mathbf{0 . 8}$} & RSS & 1.229081 & 1.207336 & 1.181370 & 1.152830 & 1.172376 & 1.169775 \\
& MRSS & 1.095716 & 1.082758 & 1.004751 & 1.077614 & 0.959124 & 0.911237 \\
& QRSS & 1.136847 & 1.146721 & 1.111260 & 1.119896 & 1.000603 & 1.072861 \\
& EORSS & 1.255472 & 1.455288 & 1.176388 & 1.338771 & 1.154653 & 1.347356 \\
\hline \multirow{4}{*}{$\mathbf{0 . 6}$} & RSS & 1.297399 & 1.296949 & 1.301181 & 1.276983 & 1.334956 & 1.287191 \\
& MRSS & 1.162400 & 1.133664 & 1.073900 & 1.122607 & 1.089163 & 1.231511 \\
& QRSS & 1.215672 & 1.231638 & 1.235270 & 1.217134 & 1.188624 & 1.179492 \\
& EORSS & 1.346461 & 1.611306 & 1.336911 & 1.522128 & 1.407576 & 1.470691 \\
\hline \multirow{2}{*}{$\mathbf{0 . 4}$} & RSS & 1.390579 & 1.393325 & 1.397054 & 1.434512 & 1.355597 & 1.372512 \\
& MRSS & 1.245353 & 1.248367 & 1.208450 & 1.248561 & 1.243356 & 1.153961 \\
& QRSS & 1.286921 & 1.342806 & 1.303176 & 1.362345 & 1.282150 & 1.238267 \\
& EORSS & 1.417525 & 1.821600 & 1.410641 & 1.792336 & 1.490825 & 1.638944 \\
\hline
\end{tabular}




\section{EVEN ORDER RANKED SET SAMPLING}

The samples of bivariate-beta and bivariate-gamma distributions are generated by using method of mixture approach. For different values of $\rho$ and $k$, the efficiency of the ratio estimators for RSS, MRSS, QRSS and suggested EORSS relating to the SRS from bivariate-beta and bivariate-gamma are computed on the basis of 60,000 repetitions and presented in Table 2 and 3 respectively.

Results for normal and non-normal distributions presented in Tables 1-3 indicate an increase in efficiency of the ratio estimators based on EORSS, RSS, MRSS and QRSS designs to the SRS ratio estimator. The estimation method EORSS is superior to the other designs considered in this study because it provides an efficient ratio estimator for estimation of the population mean of study variable. The efficiency of the ratio estimators for normal distribution is higher than that of those from beta and gamma distributions. It is also found that EORSS method shows an efficient behavior in estimating the population mean even for low correlations.

\section{Conclusion}

A novel ranked set sampling technique EORSS was proposed for precise estimation of the population mean. The sample means and ratio estimators based on EORSS along with their MSEs are derived. Both normal and non-normal distributions are considered to demonstrate the performance of the obtained EORSS estimates under ranking considering on auxiliary variable. A mixture approach was applied to simulate the non-normal bivariate distributions. A simulation process was conducted to compare the relative precision of the EORSS ratio estimators with SRS, RSS, MRSS and QRSS ratio estimators. The EORSS ratio estimates were more precise and efficient than the existing ranked set sampling ratio estimates considered for different values of $\rho$ and sample size. The EORSS scheme was equally efficient in estimating the population mean in absence of normality. Therefore, based on above mentioned findings EORSS scheme can be recommended for precise estimation of population parameters. These results can be extended to estimate the mean and regression type estimators of the population mean based on EORSS scheme.

\section{References}

Ahmad, M., Hanif, M. \& Muttlak, H. A. (2010). Ranked Set Sampling. Cambridge, UK: Cambridge Scholars Publishing. 


\section{TAYYAB ET AL.}

Arnold, B. C., Balakrishnan, N. \& Nagaraja, H. N. (1992). A first course in order statistics. NY: John Wiley \& Sons.

Bouza, Carlos N. (2010). Ranked set sampling using auxiliary variables of a randomized response procedure for estimating the mean of a sensitive quantitative character. Journal of Modern Applied Statistical Methods, 9(1), 248-254. https://doi.org/10.22237/jmasm/1272687780

Dell, T. R. \& Clutter, J. L. (1972). Ranked set sampling theory with order statistics background. Biometrics, 28(2), 545-555. https://doi.org/10.2307/2556166

Haq, A., Brown, J., Moltchanova, E. \& Al-Omari, A. I. (2014). Mixed ranked set sampling design, Journal of applied statistics, 41(10), 2141-2156.

https://doi.org/10.1080/02664763.2014.909781

Ibrahim, K. (2011). On comparison of some variation of ranked set sampling. Sains Malaysiana, 40(4), 397-401.

Khan, L. \& Shabbir, J. (2016). Hartley-Ross type unbiased estimators using ranked set sampling and stratified ranked set sampling. North Carolina Journal of Mathematics and Statistics, 2, 10-22.

Koyuncu, N. (2015). Ratio estimation of the population mean in extreme ranked set and double robust extreme ranked set sampling, Int. J. Agricult. Stat. Sci, 11(1), 21-28.

McIntyre, G. A. (1952). A method for unbiased selective sampling using ranked sets. Australian Journal of Agricultural Research, 3(4), 385-390.

https://doi.org/10.1071/ar9520385

Michael, J. R. \& Schucany, W. R. (2002). The mixture approach for simulating bivariate distributions with specific correlations. The American Statistician, 56(1), 48-54. https://doi.org/10.1198/000313002753631367

Muttlak, H. A. (1997). Median ranked set sampling. J. App. Statist. Sci., 6(4), 245255 .

Muttlak, H. A. (2003). Investigating the use of quartile ranked set samples for estimating the population mean. Applied Mathematics and Computation, 146(2-3), 437443. https://doi.org/10.1016/s0096-3003(02)00595-7

Noor-ul-Amin, M. \& Hanif, M. (2012). Some exponential estimators in survey sampling. Pakistan journal of Statistics, 28(3), 367-374.

Noor-ul-Amin, M., Javiad, A. \& Hanif, M. (2017). Estimation of population mean in systematic random sampling using auxiliary information. Journal of Statistics and Management Systems, 20(6), 1095-1106.

https://doi.org/10.1080/09720510.2017.1364500 


\section{EVEN ORDER RANKED SET SAMPLING}

Samawi, H. M. \& Muttlak, H. M. (1996). Estimation of ratio using ranked set samples. Biometrical Journal, 38(6), 753-764. https://doi.org/10.1002/bimj.4710380616

Samawi, H. M. \& Muttlak, H. M. (2000). On ratio estimation using median ranked set sampling. Journal of Applied Statistical Science, 10(2), 89-98.

Samawi, H. M. (2002). On ratio estimation using extreme ranked set samples. Abhath Al-Yarmouk, Basic Sciences and Engineering, 11, 815-827.

Sanaullah, A., Ameer Ali, H., Noor-ul-Amin, M. \& Hanif, M. (2014). Generalized exponential chain ratio estimators under stratified two-phase random sampling. Applied Mathematics and Computation, 226, 541-547. https://doi.org/10.1016/j.amc.2013.10.088

Stokes, S. L. (1977). Ranked set sampling with concomitant variables. Communication in Statistics - Theory and Methods, 6(12), 1207-1211. https://doi.org/10.1080/03610927708827563

Takahasi, K. \& Wakimoto, K. (1968). On unbiased estimates of the population mean based on the sample stratified by means of ordering. Annals of the Institute of Statistical Mathematics, 20(1), 1-31. https://doi.org/10.1007/bf02911622

Zamanzade, E. \& Al-Omari, A. I. (2015). New ranked set sampling for estimating the population mean and variance. Hacettepe Journal of Mathematics and Statistics, 46(92), 1891-1905. https://doi.org/10.15672/hjms.20159213166 